\title{
Microsaccade Control Signals in the Cerebellum
}

\author{
(DDaniel Arnstein, ${ }^{1,2}$ Marc Junker, ${ }^{1,2}$ Aleksandra Smilgin, ${ }^{1,2}$ Peter W. Dicke, ${ }^{1}$ and Peter Thier ${ }^{1}$ \\ ${ }^{1}$ Department of Cognitive Neurology, Hertie Institute for Clinical Brain Research, Tübingen 72076, Germany; and ${ }^{2}$ Graduate School of Neural and \\ Behavioural Sciences, University of Tübingen, Tübingen 72074, Germany
}

\begin{abstract}
Microsaccades, the small saccades made when we try to keep the eyes still, were once believed to be inconsequential for vision, but recent studies suggest that they can precisely relocate gaze to tiny visual targets. Because the cerebellum is necessary for motor precision, we investigated whether microsaccades may exploit this neural machinery in monkeys. Almost all vermal Purkinje cells, which provide the eye-related output of the cerebellar cortex, were found to increase or decrease their simple spike firing rate during microsaccades. At both the single-cell and population level, microsaccade-related activity was highly similar to macrosaccade-related activity and we observed a continuous representation of saccade amplitude that spanned both the macrosaccade and microsaccade domains. Our results suggest that the cerebellum's role in fine-tuning eye movements extends even to the oculomotor system's smallest saccades and add to a growing list of observations that call into question the classical categorical distinction between microsaccades and macrosaccades.
\end{abstract}

Key words: cerebellum; fixational eye movements; microsaccades; oculomotor; Purkinje cells

\section{Introduction}

In primates, high-acuity vision is limited to the fovea. To collect visual information about a larger scene, it is necessary to make saccades, rapid eye movements that bring the fovea to a new target. Because saccades are very brief, ballistic movements, online sensory feedback does not arrive soon enough to guide them, so the initial motor plan must be extremely precise (Latour, 1962; Keller and Robinson, 1971; Guthrie et al., 1983). The cerebellum is required for motor accuracy and consistency and saccade dysmetria results from a lesion to the oculomotor vermis, the eyemovement-related region of cerebellar cortex, or the fastigial oculomotor region, the part of the deep cerebellar nuclei that relays this information to other eye-movement-related structures (Ritchie, 1976; Optican and Robinson, 1980; Robinson et al., 1993; Takagi et al., 1998; Barash et al., 1999; Goffart et al., 2004; Golla et al., 2008; Ignashchenkova et al., 2009). But does the cerebellum fine-tune microsaccades as well?

Microsaccades, the small saccades made during fixation, were once considered to be inconsequential for vision because they are too small to bring a new object to the fovea. Early reports suggested that they only occur in laboratory conditions (Steinman et

Received June 16, 2014; revised Dec. 30, 2014; accepted Jan. 6, 2015.

Author contributions: D.A., M.J., A.S., P.W.D., and P.T. designed research; M.J. and A.S. performed research; D.A. analyzed data; D.A., M.J., A.S., P.W.D., and P.T. wrote the paper.

The work was supported by a Boehringer Ingelheim Fonds doctoral fellowship (to D.A.), the Marie Curie Initial Training Network (Grant PITN-GA-2009-238214; to P.T.), the German Ministry of Education, Science, Research, and Technology through the Bernstein Center for Computational Neuroscience (Grant FKZ 01GQ1002), and the Deutsche Forschungsgemeinschaft (Grant FOR 1847-A3 TH425/13-1; to P.T.). We thank Ziad Hafed for helpful comments on the manuscript.

We dedicate this paper to the memory of Dan Arnstein, our dear young colleague, who passed away unexpectedly on September 8, 2014 in the middle of the revision process of this paper and are grateful to Akshay Markanday for his invaluable help when trying to finish the revision.

The authors declare no competing financial interests.

Correspondence should be addressed to Peter Thier, Department of Cognitive Neurology, Hertie Institute for Clinical Brain Research, Hoppe-Seyler-Str. 3, 72076 Tübingen, Germany. E-mail: thier@uni-tuebingen.de.

DOI:10.1523/JNEUROSCI.2458-14.2015

Copyright $\odot 2015$ the authors $\quad 0270-6474 / 15 / 353403-09 \$ 15.00 / 0$ al., 1973), that they are not necessary for maintaining accurate fixation (Kowler and Steinman, 1980), and that they are actively suppressed during tasks that require high visual acuity (Winterson and Collewijn, 1976). However, recent evidence suggests that the preferred fixation locus is an order of magnitude smaller than the fovea (Putnam et al., 2005) and, just as macrosaccades serve to bring objects of interest to the fovea, microsaccades bring taskrelevant visual targets to the preferred subregion of the fovea (Ko et al., 2010; Poletti et al., 2013), improving visual acuity (Poletti et al., 2013). Given the need for precision in microsaccades, we hypothesized that the cerebellum may fine-tune microsaccades in the same way as it does macrosaccades.

Muscimol injection in the fastigial oculomotor region biases fixation position (Robinson et al., 1993; Goffart et al., 2004; Quinet and Goffart, 2005) and microsaccade direction (Goffart et al., 2004; Guerrasio et al., 2010), demonstrating that the output of the cerebellum can influence microsaccades. However, the nature of the cerebellar control signals remains unknown. Here, we recorded the simple spike (SS) discharge of vermal Purkinje cells (PCs) while monkeys made microsaccades and macrosaccades to test the hypothesis that these two types of movements share a common representation in the cerebellum.

\section{Materials and Methods}

Subjects, surgery, and recording methods

All animal experiments were approved by the local animal care committee and conducted in accordance with German law and the National Institutes of Health's Guide for the Care and Use of Laboratory Animals. Three adult male rhesus (Macaca mulatta) monkeys (Monkey E: 10-12 years of age, Monkey I: 6 years, and Monkey H: 10-11 years during the experiments) were subjects in this study. They were implanted with a magnetic scleral search coil to record the eye position (Judge et al., 1980), a titanium head post to painlessly immobilize the head during experiments, and a circular titanium recording chamber located over the midline of the cerebellum to allow electrophysiological recordings (Thier and Erickson, 1992). In Monkey H, the central axis of the chamber was placed 

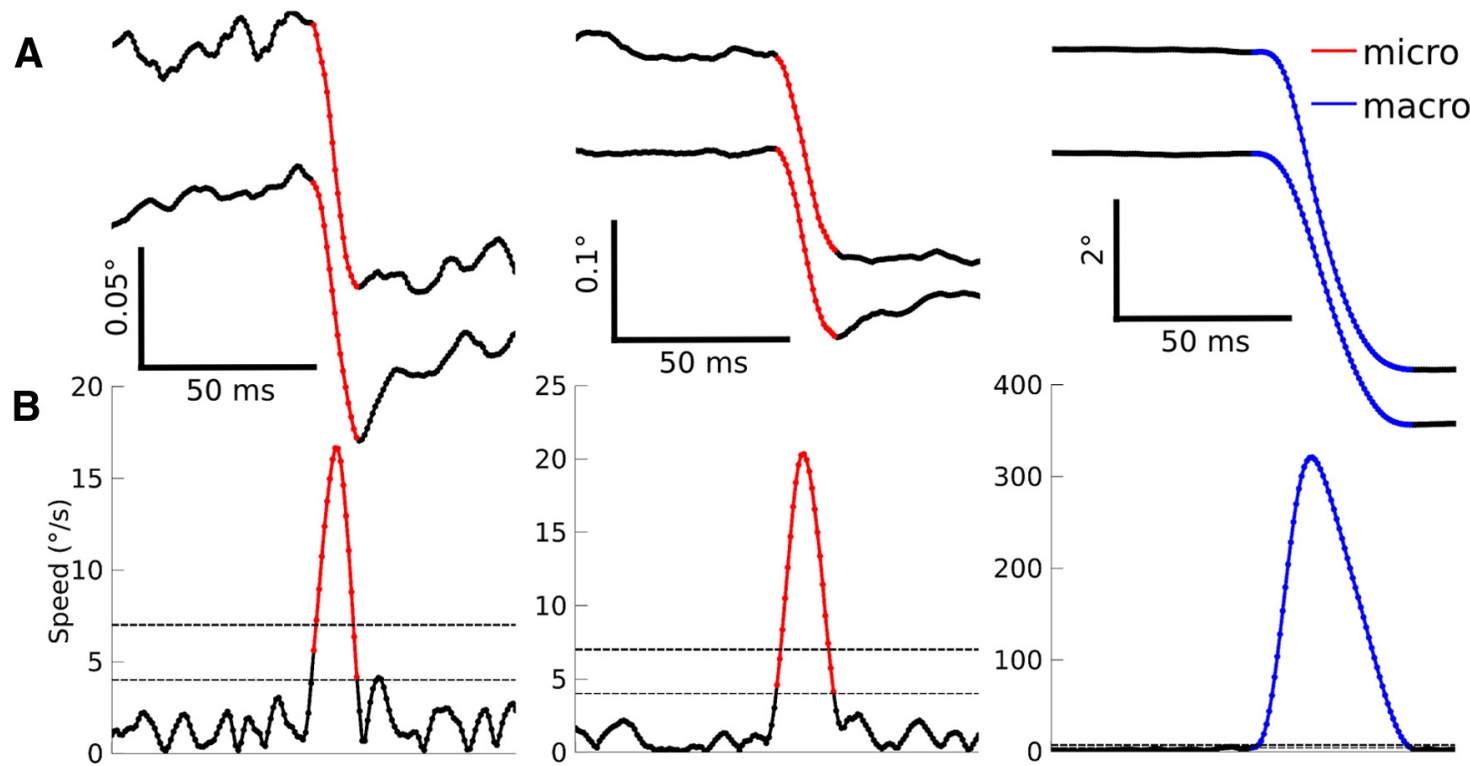

Figure 1. Saccade detection. $\boldsymbol{A}$, Horizontal eye position (top) vertical eye position (bottom) for three example saccades. $\boldsymbol{B}$, Eye speed with the lenient (thin dotted line) and stringent (thick dotted line) speed thresholds overlaid (see Materials and Methods). Detected microsaccades are shown in red and macrosaccades are shown in blue.

$5 \mathrm{~mm}$ left to the sagittal midline, tilted by $15^{\circ}$ posterior and shifted by 10 $\mathrm{mm}$ posterior with respect to the intra-aural line. In Monkey I, the chamber was placed over the sagittal midline, tilted by $40^{\circ}$ posterior with chamber axis pointing toward the intra-aural line. In Monkey E, the chamber was placed in sagittal midline tilted by $20^{\circ}$ posterior and shifted $2.9 \mathrm{~mm}$ posterior with respect to the intra-aural line. The position and orientation of the implants were carefully planned using a presurgical MRI and confirmed using a postsurgical MRI that helped to direct electrodes to the oculomotor vermis. All surgical procedures were conducted using aseptic techniques under the full anesthesia consisting of isofluorane supplemented with remifentanil $(1-2 \mu \mathrm{g} / \mathrm{kg} / \mathrm{min})$ and all relevant physiological parameters such as body temperature, heart rate, blood pressure, $\mathrm{pO}_{2}$, and $\mathrm{pCO}_{2}$ were monitored. Postoperatively, buprenorphine was given until no sign of pain was evident. Animals were allowed to fully recover before starting the experiments.

\section{Behavioral procedures}

Each monkey was trained to voluntarily come to the primate chair and remain seated with the head fixed painlessly for the duration of the experiment. During experimental sessions, the monkeys had to work for their daily fluid intake needs by complying with the behavioral demands of the experiments. Specifically, each successful eye movement trial was rewarded with water. If needed, additional fluid and/or juicy fruits were provided after experiments to satisfy the daily fluid requirements. However, the monkeys did not get water in their home cages. Usually every fortnight, they were granted a 2 day vacation with free access to water and juicy fruits in their home cages.

The monkey was head-fixed in the set-up $40 \mathrm{~cm}$ in front of a CRT monitor in complete darkness. The eye position was calibrated using a standard 9-point calibration routine (typical grid size: $30 \times 30^{\circ}$, target diameter: $0.4^{\circ}$ ) and the animal was subsequently asked to make visually guided saccades (146/146 PCs) and sometimes visually guided smooth pursuit eye movements as well (86/146 PCs). The fixation target was a white circle of $0.4^{\circ}$ diameter and the animal was considered to be fixating adequately when the eye position was within a $2^{\circ}$ or $3^{\circ}$ square fixation window centered on the stimulus. When fixation was broken, the trial was aborted and no reward was delivered. Each trial consisted of an initial fixation period on a central target $(500-1500 \mathrm{~ms})$, followed by a $10^{\circ}$ target jump or a smooth target movement with constant velocity $(10 \%)$. The direction of the target jump or smooth movement was randomly selected in each trial from $8\left(0^{\circ}, 45^{\circ}, 90^{\circ}, 135^{\circ}, 180^{\circ}, 215^{\circ}, 270^{\circ}\right.$, and $315^{\circ}$, $127 / 146$ PCs $)$ or $4\left(0^{\circ}, 90^{\circ}, 180^{\circ}\right.$, and $270^{\circ}, 19 / 146$ PCs $)$ possible directions.
All macrosaccades, including spontaneous saccades performed during the experimental sessions, were used in analysis with the exception of catch-up saccades performed during smooth pursuit. However, microsaccades were only included if they occurred during central fixation.

\section{Electrophysiological procedures}

The oculomotor vermis (lobuli VI-VII) was identified by the presurgical MRI and confirmed by electrophysiological criteria, namely, the modulation of background activity by spontaneous saccades in the dark. PCs were recorded extracellularly using glass-coated tungsten microelectrodes (0.5-2 $\mathrm{M} \Omega$ impedance; Alpha Omega). Single units were identified online by the presence of both SS and complex spikes (CS) and a depression of the SS rate for $\sim 10 \mathrm{~ms}$ after each CS, as described previously (Catz et al., 2008; Dash et al., 2013). The raw voltage trace and eye trace obtained from the scleral search coils were recorded and stored at 25 $\mathrm{kHz}$.

\section{Data analysis}

Eye position. The eye position trace was downsampled to $1000 \mathrm{~Hz}$ and filtered with a second-order Savitzky-Golay filter (frame length: $20 \mathrm{~ms}$ ). To estimate the amount of residual noise in the eye traces, we computed the SD of the horizontal and vertical eye position during fixation periods between microsaccades. This provides an upper bound estimate of the noise because true oculomotor behaviors such as drift and tremor also contribute to the variance in the eye position. The median SD of horizontal eye position was $0.010^{\circ}$ and the median SD of the vertical eye position was $0.011^{\circ}$.

Next, the horizontal and vertical components of the velocity were obtained by numerical differentiation. Saccade onset and offset were defined using a lenient $4 \%$ s speed threshold, but saccades were only included if they additionally crossed a more stringent speed threshold of $7 \%$ to reduce the number of spurious saccades detected (Fig. 1). For the 26/146 PCs recorded in sessions with noisier eye traces, $5 \%$ s and $15 \%$ were used as the lenient and stringent thresholds, respectively. Saccade direction and amplitude were computed as the direction and norm, respectively, of the vector pointing from the eye position at saccade onset to the eye position at saccade offset.

When there is high-frequency noise in the eye signal, the eye speed will appear to be high and spurious saccades may be detected. To automatically distinguish between spurious saccades and true oculomotor behavior, we implemented a variety of criteria designed to identify transient periods with anomalous oculomotor behavior and high levels of noise in the eye trace. In particular, we excluded saccades for which the peak 

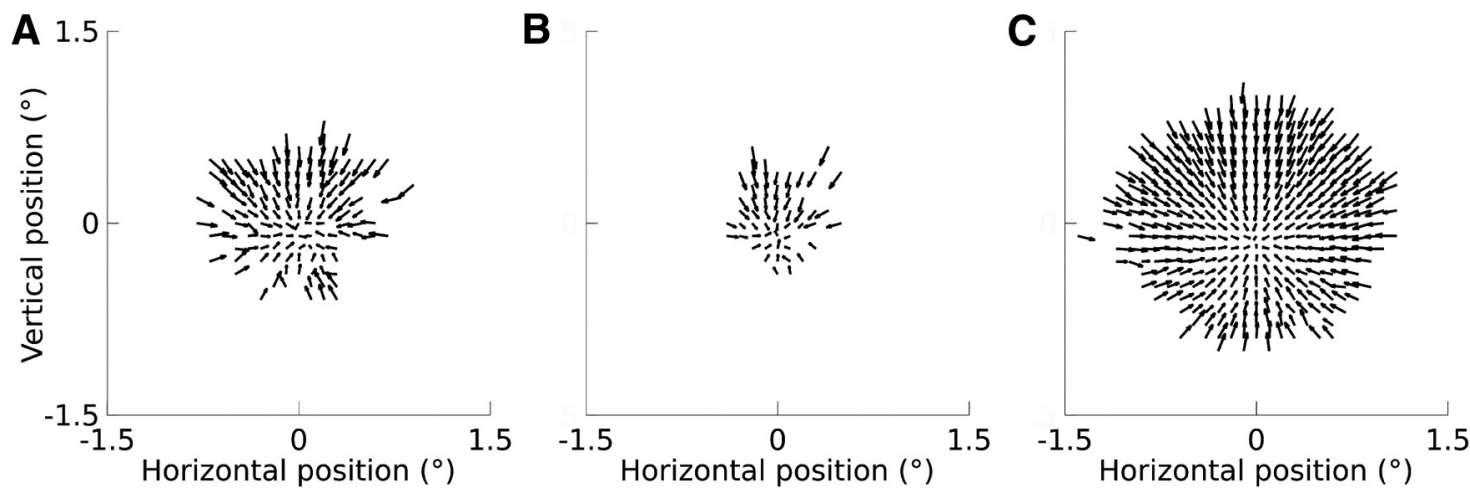

Figure 2. Relationship between eye position and microsaccade direction and amplitude for two example sessions shown in $\boldsymbol{A}$ and $\boldsymbol{B}(n=1415$ microsaccades $(\boldsymbol{A}), n=616$ microsaccades $(\boldsymbol{B})$, and all sessions $(C)\left(n=36812\right.$ microsaccades). Arrows originate from the eye position at the time of saccade onset (bin size: $\left.0.1^{\circ}\right)$ and point in the direction of the median eye position at the time of saccade offset. Amplitudes are represented as $20 \%$ of their true length for visual clarity. Starting positions were excluded if fewer than three microsaccades originated from that position for example sessions and $<15$ microsaccades for all sessions because far more microsaccades were available.

speed in a $40 \mathrm{~ms}$ window before the saccade exceeded $80 \%$ of the saccade peak speed, saccades for which the eye displacement between $30 \mathrm{~ms}$ before onset and $30 \mathrm{~ms}$ after offset was $>130 \%$ of the saccade amplitude and saccades containing multiple crossings of the stringent speed threshold connected by a bridge in which the eye speed never fell below the more lenient threshold. Any remaining spurious or malformed saccades were identified manually and excluded.

Microsaccades were distinguished from macrosaccades using an amplitude threshold of $1^{\circ}$ (Martinez-Conde et al., 2013) and the median microsaccade amplitude was $0.65^{\circ}\left(\mathrm{M} 1: 0.71^{\circ}, \mathrm{M} 2: 0.65^{\circ}, \mathrm{M} 3: 0.62^{\circ}\right)$. This is larger than in most studies, although there is also considerable variability between the average microsaccade amplitudes described in past reports, which include $0.8^{\circ}$ (Bair and O'Keefe, 1998), $0.73^{\circ}$ (Guerrasio et al., 2010), $0.67^{\circ}$ (Snodderly et al., 2001), $0.46^{\circ}$ (Otero-Millan et al., 2011), $0.33^{\circ}$ (Ko et al., 2010; Poletti and Rucci, 2010), and $0.23^{\circ}$ (Hafed et al., 2009).

Microsaccades were excluded unless they were initiated while the monkey was fixating within a square window of $3^{\circ}$ centered on the central fixation target. In practice, most microsaccades were initiated while the eye eccentricity was considerably lower than the limits imposed by the fixation window (median horizontal eccentricity: $0.31^{\circ}$, vertical: $0.30^{\circ}$ ). If at least 50 microsaccades and 50 macrosaccades remained in the session after exclusion, then the session was included in neuronal analyses, but most sessions contained far more microsaccades (median $=198, \mathrm{Q} 1=138, \mathrm{Q} 3=457)$ and macrosaccades (median = $315.5, \mathrm{Q} 1=258, \mathrm{Q} 3=1397)$. For analyses of saccade direction and amplitude selectivity, a minimum of five saccades per direction or amplitude was required.

As in past reports (Cornsweet, 1956; Guerrasio et al., 2010), microsaccades were typically corrective, bringing the line of gaze toward the fixation target (Fig. 2).

Electrophysiology. SS times were aligned to the onset of saccades and the spike density function was obtained by convolving the spike trains with a Gaussian $(\sigma=10 \mathrm{~ms})$. PCs were tested for saccade-related activity by comparing the mean spike density between saccade onset and offset to the mean spike density in the baseline period: $[-200,-100]$ ms relative to saccade onset (Wilcoxon signed-rank tests with $\alpha=0.05$ ). According to this criterion, 146/167 PCs showed saccade-related activity for either microsaccades or macrosaccades and all further analyses were performed only for these 146 saccade-related PCs.

Saccade-related PCs show highly heterogeneous spike rate changes during saccades. Some PCs increase their spike rate during saccades and others decrease their spike rate. The precise timing also varies across cells. Moreover, individual PCs often have a preferred saccade direction and amplitude for which they respond with a larger change in spike rate change than they do for saccades of other directions and amplitudes. Further analyses were designed to characterize these idiosyncratic features and test whether they were shared or distinct between microsaccades and macrosaccades.
For PC lag analyses, the perisaccade time was defined as $[-25,100] \mathrm{ms}$ relative to saccade onset and $[-50,75]$ ms relative to the time of saccade peak velocity. For microsaccades and macrosaccades separately, we computed the neuronal lag as the perisaccade time associated with the highest spike rate for bursting cells or lowest spike rate for pausing cells. We then tested whether the lag was correlated between microsaccades and macrosaccades using a permutation test. Briefly, the difference between the lags for microsaccades and macrosaccades was calculated for each cell, and the interquartile range (IQR) of the resulting distribution of difference scores was computed. We used the IQR as the test statistic to measure whether the difference scores were narrowly distributed, as one would expect if there were tight correlations between the lags for microsaccades and macrosaccades, or broadly distributed, as one would expect if there were no correlation between the two lags. We next shuffled which cells were associated with which macrosaccade lags and again calculated the IQR of the difference score distribution. This procedure was repeated 1000 times to obtain a null distribution of IQRs expected by chance when there is no correlation between the lag during microsaccades versus macrosaccades. We then computed the mean and SD of the null distribution and used these statistics to calculate the probability that we would obtain an IQR as small or smaller than the experimental IQR. If there were no correlation between the PC lag during microsaccades and the lag during macrosaccades, then the IQR of the true difference scores would be about as large as the typical IQRs obtained from the shuffling procedure.

We extracted direction-tuning functions for microsaccades and macrosaccades separately by computing the mean spike rate as a function of saccade direction and then compared the two tuning curves with each other using circular cross-correlation. The circular cross-correlation function was computed by taking the product of the baseline-corrected (i.e., the spike rate minus the average discharge in the baseline period as defined in the Materials and Methods section) macrosaccade direction tuning function and the baseline-corrected microsaccade direction tuning function for all possible angular lags. The optimal lag was considered to be the one associated with the largest product. Circular statistics were calculated using the circ_stat toolbox for MATLAB (Berens, 2009).

To investigate the amplitude selectivity of saccade-related responses, we binned the saccades into 9 amplitude bins, $0-0.4^{\circ}, 0.4-0.7^{\circ}, 0.7-1^{\circ}$, $1-2^{\circ}, 2-3^{\circ}, 3-5^{\circ}, 5-8^{\circ}, 8-11^{\circ}, 11-15^{\circ}$, and the amplitude tuning curves were computed as the mean spike rate during saccades of each amplitude minus the baseline spike rate. The population amplitude tuning curves were fit to an exponential function as follows:

$$
R(d)=a+\left(R_{0}-a\right) * e^{-b * d}
$$

where $R(d)$ is the baseline-corrected spike rate for the amplitude bin that is $d$ bins away from the preferred amplitude and $R_{0}$ is the baselinecorrected spike rate for the preferred amplitude. The parameters $a$ and $b$ were optimized such that the mean squared error between the data and exponential fit was minimized. When computing the mean squared er- 
ror, each amplitude distance $d$ was weighted according to the inverse of the SE to give the most weight to the most reliable measurements. This fitting procedure was performed separately for the saccade amplitude bin ranges $[-7,0]$ and $[0,7]$ (with bin 0 representing the preferred amplitude) to allow a comparison of spike rate changes during saccades that were smaller than the preferred amplitude versus larger than the preferred amplitude. Because saccades were divided into nine amplitude bins, the bin domain of the population tuning curves involved a range of eight bins, either left or right of the bin representing the preferred amplitude. However, only a range of $[-7,7]$ was considered in the analysis and the outermost bins excluded as finding the preferred amplitude in the smallest/largest amplitude bin would in many cases be a consequence of a boundary effect; that is, the erroneous assignment of the preferred amplitude to this bin just because no smaller/larger amplitudes were tested.

The exponential fits over the saccade amplitude bin ranges $[-7,0]$ and $[0,7]$ were compared using permutation tests. First, the difference scores $a_{\text {left }}-a_{\text {right }}$ and $b_{\text {left }}-b_{\text {right }}$ were computed. Next, each cell was randomly assigned to either maintain its original amplitude tuning or to mirror it relative to the preferred amplitude bin. In other words, for cells assigned to mirror their tuning function, the firing rate for saccades bigger than the preferred amplitude was switched with the firing rate for saccades smaller than the preferred amplitude. The resulting population tuning curves were again fit with exponential functions, and $a_{\text {left }}-a_{\text {right }}$ and $b_{\text {left }}-b_{\text {right }}$ were computed. This shuffling procedure was repeated 1000 times to obtain a distribution of difference scores expected by chance when there is no true difference between the fit over $[-7,0]$ and $[0,7]$. The true difference scores were then compared with this distribution to obtain $p$-values.

\section{Results}

We analyzed the SS firing rates of 146 eye-related PCs recorded in the oculomotor vermis of the cerebellum while monkeys made visually guided macrosaccades $\left(>1^{\circ}\right.$ saccades $)$ and microsaccades $\left(<1^{\circ}\right.$ saccades made during fixation). As in previous reports (Sato and Noda, 1992; Ohtsuka and Noda, 1995), the vast majority increased or decreased their spike rate during macrosaccades compared with the presaccade baseline (126/146, 86\%). Almost as many PCs changed their spike rate during microsaccades $(119 / 146,82 \%)$.

\section{Comparison of PC discharge during microsaccades versus macrosaccades}

We investigated whether the activity during microsaccades was similar to the activity during macrosaccades (Fig. 3). Although the size and duration of the SS rate changes were often quantitatively different between microsaccades and macrosaccades, the changes were qualitatively similar. To quantify the similarity, we first used the sign of the change. Neurons with a significant burst (spike rate increase) during macrosaccades $(n=57)$ usually also responded with a significant burst for microsaccades (39/57 PCs, $68 \%$, Fig. $3 A, B)$, whereas a much smaller percentage had a significant pause (spike rate decrease; $7 / 57$ PCs, $12 \%$ ) or no response (11/57 PCs, 19\%). Likewise, neurons with a significant pause during macrosaccades $(n=69)$ usually also responded with a significant pause for microsaccades (52/69 PCs, 75\%, Fig. $3 C, D)$, whereas a much smaller fraction had a significant burst $(1 / 69,1 \%)$ or no response $(16 / 69,23 \%)$. For neurons with a significant change during both microsaccades and macrosaccades $(n=99)$, the sign of the spike rate change was significantly correlated across saccade types $\left(\chi^{2}\right.$ test, $\left.p<10^{-15}\right)$. Because the sign of the change was typically the same for microsaccades and macrosaccades, for further analyses, we labeled PCs as "bursting" or "pausing" on the basis of their response to pooled saccades of all amplitudes without making a distinction between microsaccades and macrosaccades.
We next analyzed the timing of the saccade-related activity in the PCs with significant spike rate changes for both saccade types $(n=99)$. We computed the neuronal lag as the perisaccade time associated with the highest spike rate for bursting cells or lowest spike rate for pausing cells. There were substantial differences between the characteristic lags of individual PCs, which we exploited to determine whether the PC lag during macrosaccades predicted the PC lag during microsaccades. This similarity was much stronger than chance (permutation test, $p<10^{-12}$; Fig. $4 A$ ). Interestingly, most cells fell slightly below the identity line, indicating that the PC lag from saccade onset was slightly longer for macrosaccades (median $=23 \mathrm{~ms}$ ) than microsaccades (median $=17 \mathrm{~ms}$, Wilcoxon signed-rank test, $p<10^{-4}$ ). We therefore wondered whether the peak activity might be time-locked to the saccade peak speed, which occurred later for macrosaccades $($ median $=17 \mathrm{~ms})$ than microsaccades $($ median $=12 \mathrm{~ms})$, rather than saccade onset. To test this hypothesis, we realigned all spikes to the time of saccade peak speed (Fig. 4B). After the realignment, cells fell much more symmetrically around the identity line and no significant difference could be measured between the latencies, which occurred just after the peak speed (macrosaccade median $=5 \mathrm{~ms}$, microsaccade median $=5 \mathrm{~ms}$, Wilcoxon signedrank test, $p=0.23$ ). PC latencies were therefore more closely tied to the time of peak saccade speed than saccade onset and this relationship held for both microsaccades and macrosaccades.

Although the activity of most PCs was similar in sign and timing during microsaccades and macrosaccades, the direction tuning curves could nonetheless differ. To determine whether the directional preferences were similar for microsaccades and macrosaccades, we binned saccade directions into either $4\left(0^{\circ}, 90^{\circ}\right.$, $180^{\circ}$, and $\left.270^{\circ}\right)$ or 8 classes $\left(0^{\circ}, 45^{\circ}, 90^{\circ}, 135^{\circ}, 180^{\circ}, 215^{\circ}, 270^{\circ}\right.$, and $315^{\circ}$ ) depending on whether the monkey was prompted to make saccades in 4 or 8 directions. The direction selectivity curve was then computed separately for microsaccades and macrosaccades as the mean spike rate during saccades in each direction minus the baseline spike rate. Of all the PCs measured for a sufficient number of trials per saccade direction, most showed significant preferences for the macrosaccade direction (82/106 PCs, $77 \%$, Kruskal-Wallis tests with $\alpha=0.05$ ) and microsaccade direction (55/106 PCs, 52\%). For the PCs with significant direction selectivity for both microsaccades and macrosaccades $(n=48)$, the direction tuning curves were strikingly similar, showing only a quantitative, not qualitative, difference between the saccade types (Fig. 5A-C). After computing the angular distance between the two tuning curves using circular cross-correlation (see Materials and Methods), we observed that many PCs had similar direction selectivity for microsaccades and macrosaccades, preferring the same saccade direction regardless of whether the amplitude of the saccade was in the macrosaccade or microsaccade range. In Figure $5 D$, many PCs have $0^{\circ}$ angular distance between the 2 tuning functions, indicating that they have identical direction selectivity for microsaccades and macrosaccades. The distribution of angular distances between the two tuning functions was significantly different from a circular uniform distribution ( $p<0.01$, Rayleigh test).

\section{Amplitude selectivity}

The microsaccade- and macrosaccade-related discharge of PCs was similar with respect to sign of the spike rate change, the lag of the change, and direction selectivity, but there were subtle differences in spike rate between microsaccades and macrosaccades (Fig. 3). To investigate this amplitude selectivity, we binned the saccades into 9 amplitude bins, $0-0.4^{\circ}, 0.4-0.7^{\circ}, 0.7-1^{\circ}, 1-2^{\circ}$, $2-3^{\circ}, 3-5^{\circ}, 5-8^{\circ}, 8-11^{\circ}, 11-15^{\circ}$, and the amplitude tuning curves 
A
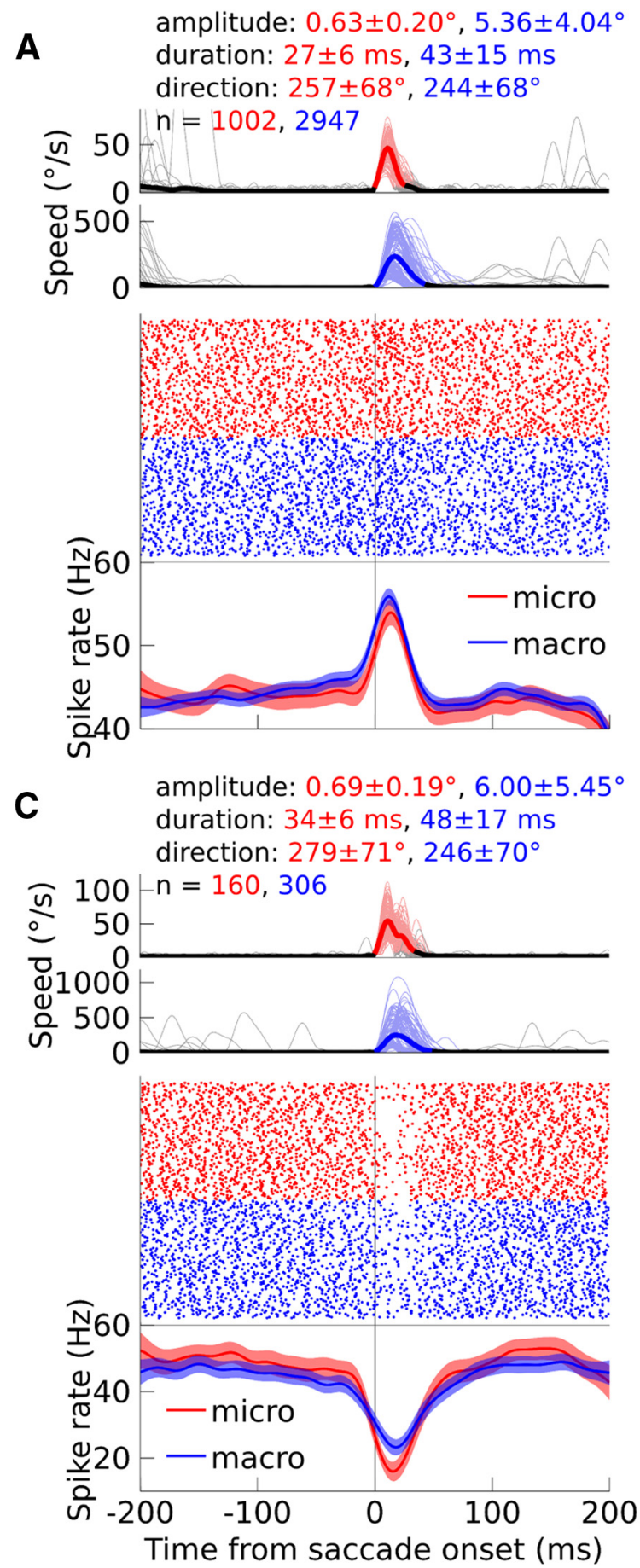

B

amplitude: $0.72 \pm 0.18^{\circ}, 6.23 \pm 4.13^{\circ}$

B duration: $31 \pm 6 \mathrm{~ms}, 48 \pm 13 \mathrm{~ms}$

direction: $237 \pm 63^{\circ}, 273 \pm 71^{\circ}$

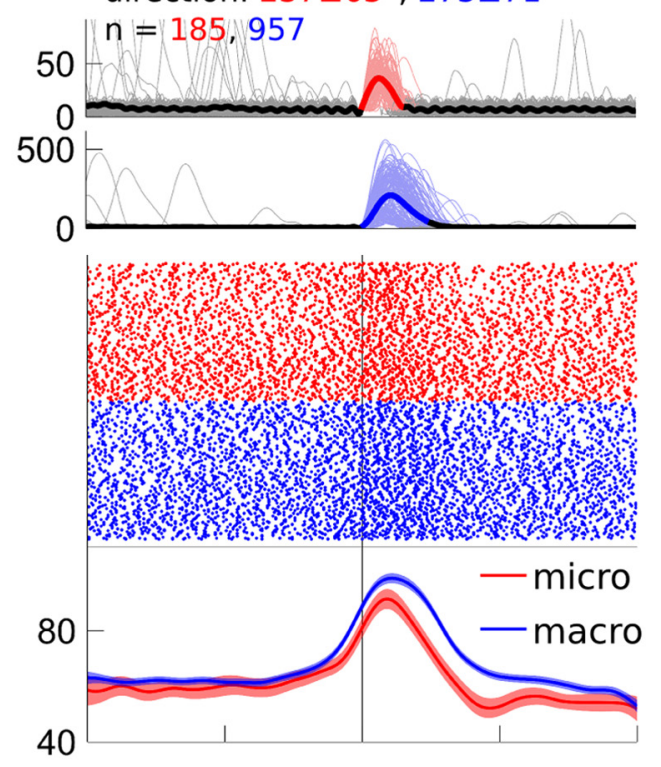

D

amplitude: $0.62 \pm 0.22^{\circ}, 6.47 \pm 4.39^{\circ}$

duration: $28 \pm 5 \mathrm{~ms}, 43 \pm 12 \mathrm{~ms}$

direction: $268 \pm 66^{\circ}, 278 \pm 65^{\circ}$
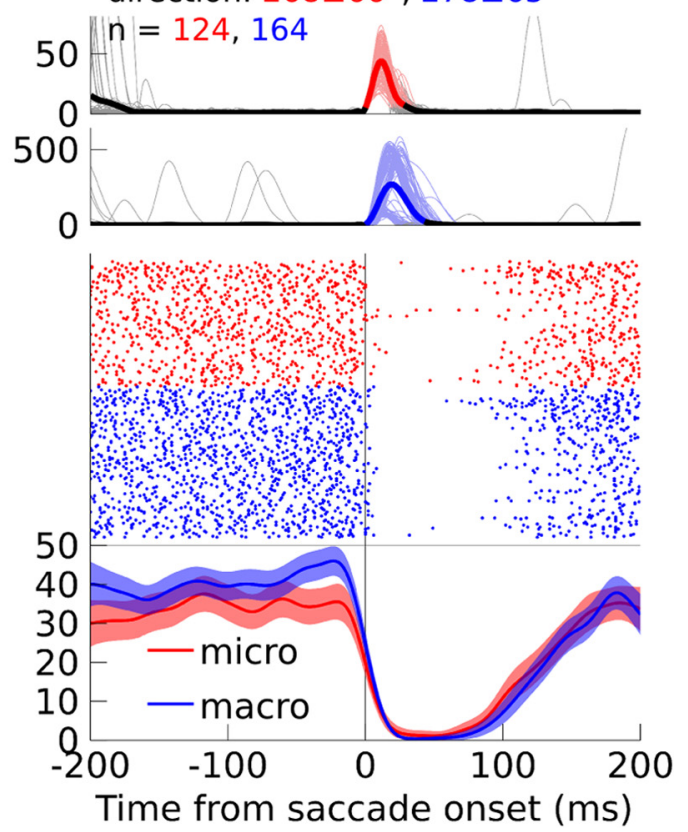

Figure 3. $\boldsymbol{A}-\boldsymbol{D}$, Examples of individual PC SS units, in each case tested for microsaccades and macrosaccades. $\boldsymbol{A}, \boldsymbol{B}$, Example bursting PCs. $\boldsymbol{C}, \boldsymbol{D}$, pausing PCs. Top, Average eye speed is shown with a thick, dark line and individual saccades are shown with thin, pale lines. The traces are colored in red (microsaccades) or blue (macrosaccades) between the onset and offset of the saccades. In addition, the mean and SD of the saccade amplitude and duration are provided, as well as the circular mean and circular SD of the direction. Finally, the number of recorded microsaccades and macrosaccades is indicated. In the middle row, the activity of the PC is shown in a raster plot, with each dot representing the time of a SS. Bottom, Spike density functions are shown with dark lines and the $95 \%$ confidence intervals are shown with light transparencies. Activity during microsaccades is shown in red and activity during macrosaccades is shown in blue. The eye traces and neuronal data are aligned to saccade onset. For both the eye traces and the raster plots, a maximum of 150 microsaccades and 150 macrosaccades are shown per panel to improve visibility. When more saccades were measured, the eye traces and raster plots depict the same random samples of 150 microsaccades and macrosaccades.

were computed as the mean spike rate during saccades of each amplitude minus the baseline spike rate. If the PC exhibited a direction preference for microsaccades and macrosaccades or at least for a certain range of saccade amplitudes, these computations were performed for this direction. In the absence of direction selectivity, one and the same direction bin, chosen at random, was considered for all amplitudes. For the PCs measured for a sufficient number of trials per saccade amplitude, most were selective for amplitude (81/103 PCs, 79\%, KruskalWallis tests with $\alpha=0.05$ ). For bursting neurons, the optimal amplitude was considered to be the one associated with the highest spike rate, whereas for pausing neurons, the optimal 
A

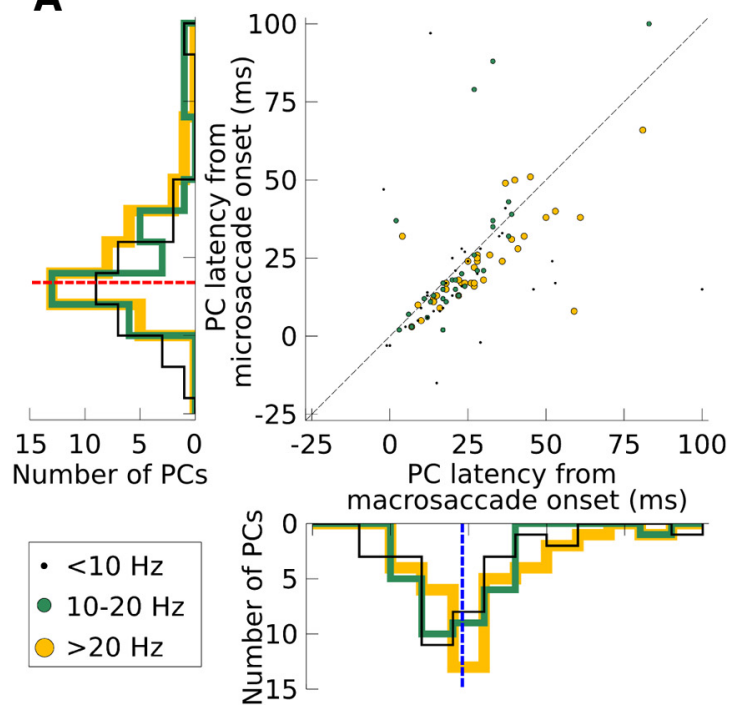

B

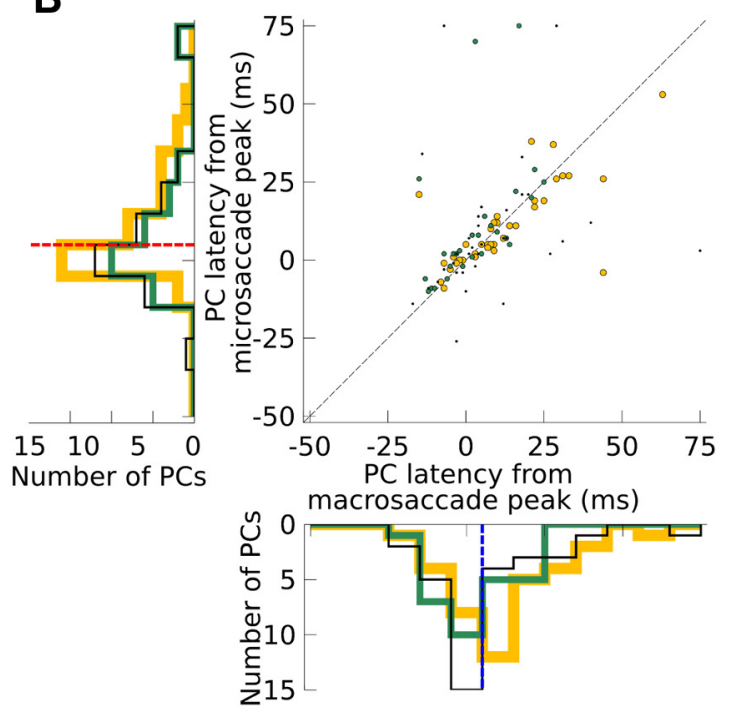

Figure 4. $\quad P C$ lag relative to saccade onset $(\boldsymbol{A})$ and saccade peak $(\boldsymbol{B})$. All $P C$ s with a significant spike rate change for both microsaccades and macrosaccades are plotted. $P(s$ with $>20 \mathrm{~Hz}$ spike rate changes for both microsaccades and macrosaccades are shown in yellow $(n=37), P(s$ with $10-20 \mathrm{~Hz}$ spike rate change are shown in green ( $n=28)$, and $\mathrm{PC}$ with $<10 \mathrm{~Hz}$ spike rate changes are shown in black $(n=34)$. For visual clarity, the dots are scaled three times larger in the legend. For comparison, the identity line $(y=x)$ is shown in black. Data are collapsed to $1 \mathrm{D}$ histograms beside each axis and medians are shown in the histograms with a red dotted line for microsaccades and blue dotted line for macrosaccades.

amplitude was associated with the lowest spike rate. While most amplitudeselective PCs preferred macrosaccades, some PCs were selective for microsaccades (10/39 bursting PCs, 26\%; 9/42 pausing PCs, 21\%). For example, the pausing PC shown in Figure $6 A$ decreased its spike rate maximally during $2-3^{\circ}$ macrosaccades, whereas the pausing PC shown in Figure $6 B$ decreased its spike rate maximally for $<0.4^{\circ}$ microsaccades. The amplitude bin that was most often preferred by PCs was $11-15^{\circ}$ (Fig. 6C), but this was most likely a boundary effect: any neuron selective for $>15^{\circ}$ saccades would be mislabeled as preferring $11-15^{\circ}$ saccades because this was the largest amplitude tested.

We next aligned the tuning curves of amplitude-tuned PCs to the preferred amplitude and fit an exponential function to the resulting population tuning curves (Fig. $6 D)$. The left and right sides of the tuning curve were fit independently. For both bursting and pausing cells, no significant difference could be observed between the tuning curve over the domain $[-7,0]$ and the tuning curve over the domain $[0,7]$ (permutation tests: all $p>0.1$; see Materials and Methods). This symmetry about the line $x=0$ indicates that the behavior of microsaccade-tuned PCs during macrosaccades was similar to the behavior of macrosaccade-tuned PCs during microsaccades. The size of the spike rate change decreased with increasing distance from the cell's preferred amplitude regardless of whether the saccade was bigger or smaller than the preferred amplitude.
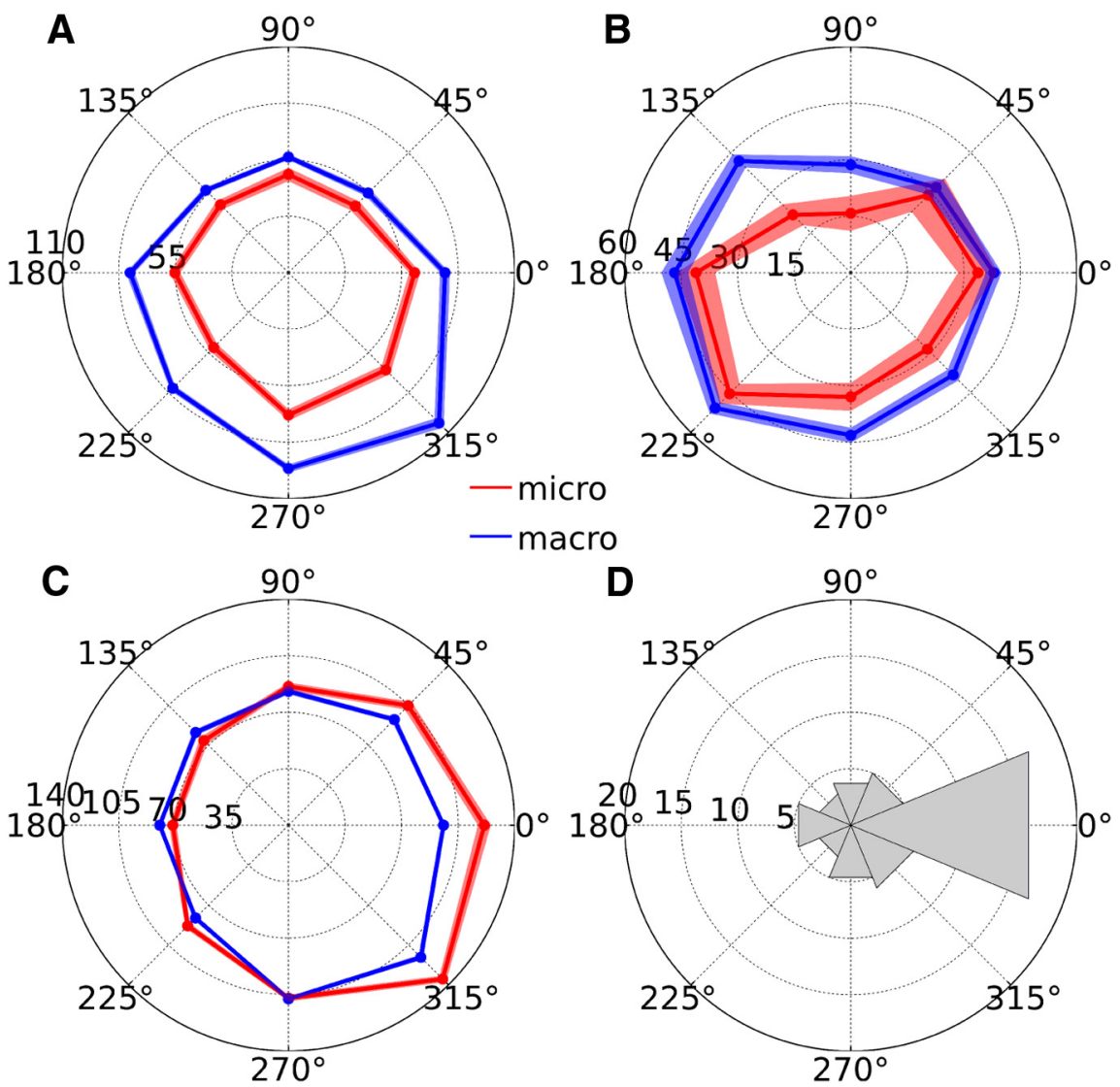

Figure 5. Direction selectivity. $\boldsymbol{A}-\boldsymbol{C}$, Direction tuning curves of three example PCS, with microsaccade-related activity shown in red and macrosaccade-related activity shown in blue. The angle of the polar plot corresponds to the saccade direction and the radius corresponds to the spike rate. Light transparencies indicate \pm 1 SEM. $D$, Circular histogram of the angular distance between the microsaccade-related and macrosaccade-related direction tuning curves, computed for each $P C(n=48) ; 0^{\circ}$ indicates perfect correspondence between the two tuning curves, $180^{\circ}$ indicates opposite selectivity, $45-135^{\circ}$ indicate counterclockwise shifts of the microsaccade-related direction tuning curve relative to the macrosaccade-related direction tuning curve, and $225-315^{\circ}$ indicate clockwise shifts of the microsaccade-related direction tuning curve relative to the macrosaccade-related direction tuning curve. 

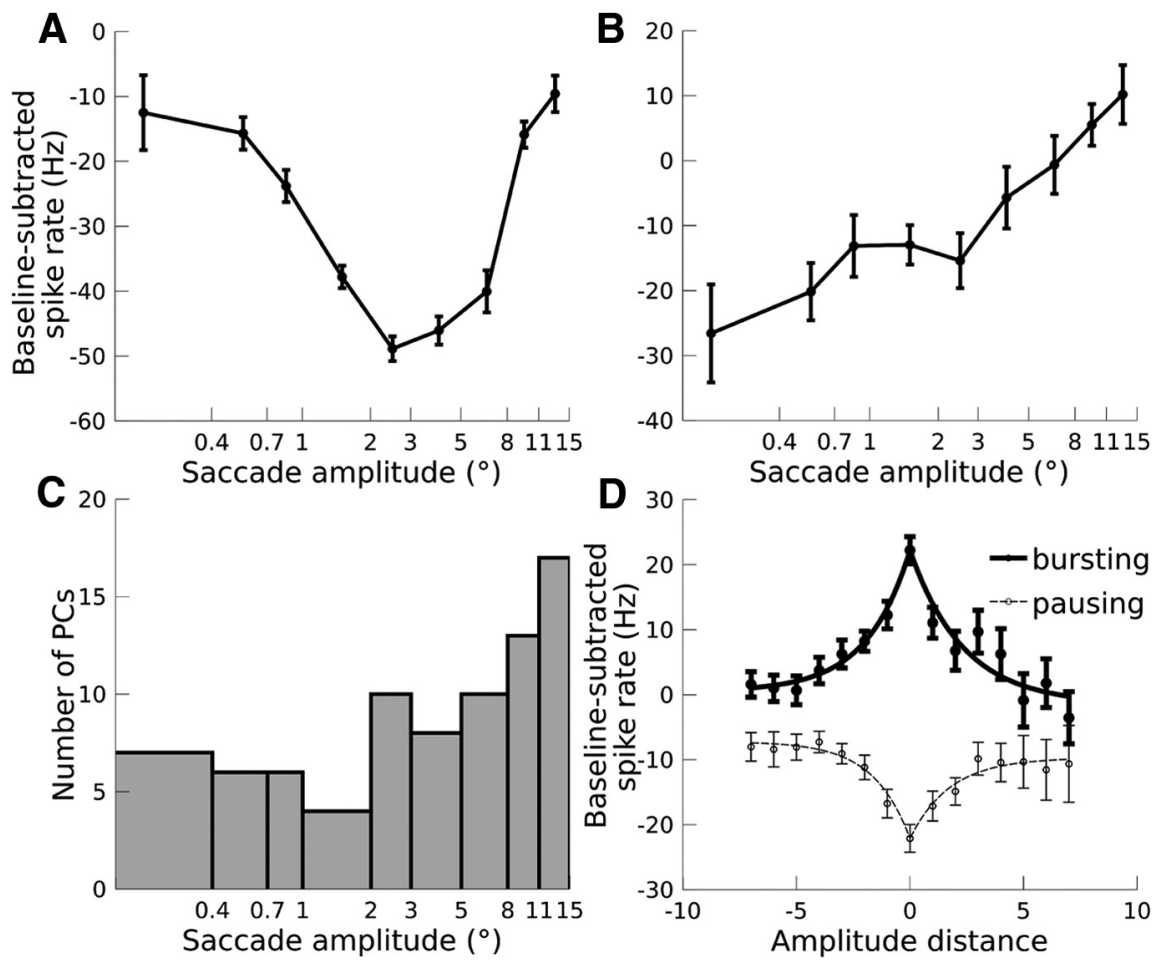

Figure 6. Amplitude selectivity. $\boldsymbol{A}$, Example pausing PC tuned for macrosaccades. $\boldsymbol{B}$, Example pausing PC tuned for microsaccades. $\boldsymbol{C}$, Histogram of preferred amplitudes for all amplitude-selective PCs $(n=81)$. For $\boldsymbol{A}-\boldsymbol{C}$, the $\boldsymbol{x}$-axis is scaled logarithmically. $D$, Population tuning curves. Spike rate is plotted against distance from the preferred amplitude, with positive amplitude distances corresponding to saccades larger than the preferred amplitude and negative amplitude distances corresponding to saccades smaller than the preferred amplitude. Population tuning curves are shown separately for bursting PCs (thick line, $n=39$ ) and pausing PCs (dotted line, $n=42$ ). Error bars indicate \pm 1 SEM. Note that the error bars are larger for positive amplitude distances than negative amplitude distances because most $P C s$ were tuned for macrosaccades, leaving fewer $P C s$ to contribute to the portion of the tuning curve corresponding to positive amplitude distances.
A

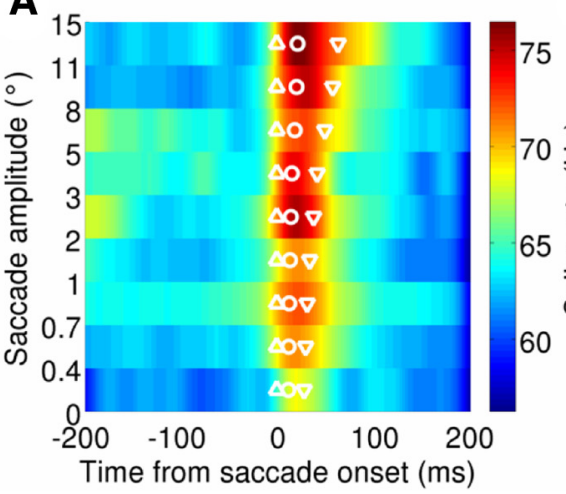

B

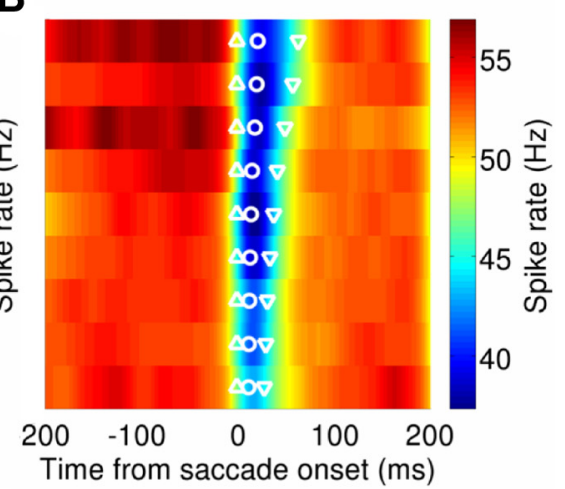

Figure 7. Population activity for bursting $\mathrm{PCs}(\boldsymbol{A} ; n=48)$ and pausing $\mathrm{PCs}(\boldsymbol{B} ; n=55)$. Spike density functions are separated according to the saccade amplitude, with microsaccades shown in the bottom three rows. The population average spike rate is shown with the color map. For each amplitude, the mean saccade onset, time of peak speed, and offset are shown with the upward triangle, circle, and downward triangle, respectively.

\section{Population spike rate}

Previous studies have shown that the saccade-related response averaged across larger populations of oculomotor vermal PCs ends when the saccade ends (Thier et al., 2000; Catz et al., 2008). To determine whether this relationship extends to the domain of microsaccades as well, we averaged the activity across PCs for saccades of varying amplitudes and therefore varying durations (Fig. 7). Saccades in all directions were included in this analysis. Although past reports (Thier et al., 2000; Catz et al., 2008) concen- trated primarily on bursting PCs, we analyzed bursting and pausing PCs separately because we recorded similar numbers of bursting versus pausing PCs. For all amplitudes, the spike rate was significantly higher during saccades than baseline for bursting PCs and significantly lower than baseline for pausing PCs (paired $t$ tests with $\alpha=0.05$ ). Even the smallest microsaccades evoked a significant spike rate change at the population level, and the sign of this change was the same across all amplitudes.

To estimate the duration of the population response, we used an arbitrary response threshold of the baseline spike rate plus $5 \mathrm{~Hz}$ for bursting PCs or minus $5 \mathrm{~Hz}$ for pausing PCs. As in past reports (Thier et al., 2000; Catz et al., 2008), the duration of the population PC activity correlated with saccade duration. This correlation held for both bursting neurons $(r=0.80$, $p<0.01)$ and pausing neurons $(r=0.90$, $p<0.001)$. Importantly, the population activity for microsaccades was similar to the population activity for macrosaccades but shorter in duration, reflecting the shorter duration of microsaccades compared with macrosaccades.

\section{Discussion}

We investigated the contribution of the cerebellum to the control of microsaccades. PCs in the oculomotor vermis showed robust microsaccade-related activity that was similar to their macrosaccade-related activity with respect to sign, lag, and direction selectivity. Although most PCs had a preferred saccade amplitude, the preferred amplitudes ranged across PCs from the smallest microsaccades to the largest macrosaccades. Moreover, the activity of microsaccade-selective neurons during macrosaccades was similar to the activity of macrosaccade-selective neurons during microsaccades, indicating a continuous representation of amplitude in the cerebellum. These results dovetail nicely with recent work showing that muscimol injection to the fastigial oculomotor region, the output structure of the oculomotor vermis (Yamada and Noda, 1987; Noda et al., 1990), biases the endpoints of both microsaccades and macrosaccades to ipsilesional space (Goffart et al., 2004; Guerrasio et al., 2010) and a growing body of research suggesting that the functions of the oculomotor control system are similar for microsaccades and macrosaccades (Van Gisbergen et al., 1981; Brien et al., 2009; Hafed et al., 2009; Ko et al., 2010; Hafed, 2011; Hafed and Krauzlis, 2012; Van Horn and Cullen, 2012; Poletti et al., 2013). We conclude that the cerebellum's role in the control of saccades extends even to the oculomotor system's smallest saccades, which occur during fixation. 
The population activity of PCs in the oculomotor vermis of monkeys lasts for the same duration as the eye movement (Thier et al., 2000) and changes in parallel to motor learning (Catz et al., 2008). Moreover, optogenetic studies in mice have demonstrated a causal link between the duration of PC population activity and movement duration (Heiney et al., 2014). Together, these results suggest that subtle changes in motor output can be achieved by fine-tuning the duration of PC activity in the cerebellum. If the population activity of the oculomotor vermis is lengthened, for example, the eyes will make a longer-duration saccade due to the monotonic relationship between saccade duration and amplitude. We observed changes in the PC population spike rate during microsaccades in addition to macrosaccades. Moreover, the population activity was sustained for a shorter duration for microsaccades, reflecting the shorter duration of the movements themselves. These results suggest that the temporal control of saccades by the cerebellum is likely to fine-tune microsaccades in addition to macrosaccades.

Our results may help to resolve a long-standing puzzle in oculomotor anatomy: the substantial projections from the fastigial oculomotor region to the superior colliculus terminate largely in the more rostral parts of the colliculus (May et al., 1990), including the rostral pole, once believed to be confined to stable fixation and not to contribute to saccades. It has been shown that recently this collicular zone also triggers microsaccades (Hafed et al., 2009; Hafed and Krauzlis, 2012). Saccade amplitude is represented along the caudal-rostral axis, with macrosaccades represented in the caudal sector and microsaccades represented in the rostral pole of the superior colliculus. The projection from the fastigial oculomotor region to the superior colliculus seems perfectly suited to allow the cerebellum to influence the collicular representations of microsaccades and macrosaccades. Our demonstration that vermal PCs offer information on both microsaccades and macrosaccades is consistent with the idea that the collicular representations of saccades are under cerebellar control regardless of amplitude. Oculomotor PCs, both at the single-cell and the population level, cover the whole range of saccade amplitudes. In other words, the oculomotor vermis uses anatomically integrated control signals rather than separate lines for saccades of different amplitudes that could be easily associated with the collicular map of saccade amplitudes. Understanding how this integrated vermal outflow is mapped onto the topography of the superior colliculus will be key to understanding how the cerebello-fastigio-collicular projection may contribute to ensuring precise saccades.

Recent evidence suggests that there is a subregion of the fovea with ultra-high visual acuity (Poletti et al., 2013). To bring an object of interest directly to this subregion, it is necessary to make microsaccades that must be precise to within just a few arcmin. Therefore, our demonstration of microsaccadic control signals in the oculomotor vermis suggests that the cerebellum may provide exactly the neural machinery that is necessary to make these eye movements so precise.

\section{References}

Bair W, O'Keefe LP (1998) The influence of fixational eye movements on the response of neurons in area MT of the macaque. Vis Neurosci 15:779786. Medline

Barash S, Melikyan A, Sivakov A, Zhang M, Glickstein M, Thier P (1999) Saccadic dysmetria and adaptation after lesions of the cerebellar cortex. J Neurosci 19:10931-10939. Medline

Berens P (2009) CircStat: a MATLAB toolbox for circular statistics. J Stat Softw 31:1-21.

Brien DC, Corneil BD, Fecteau JH, Bell AH, Munoz DP (2009) The behav- ioural and neurophysiological modulation of microsaccades in monkeys. Journal of Eye Movement Research 3:1-12.

Catz N, Dicke PW, Thier P (2008) Cerebellar-dependent motor learning is based on pruning a Purkinje cell population response. Proc Natl Acad Sci U S A 105:7309-7314. CrossRef Medline

Cornsweet TN (1956) Determination of the stimuli for involuntary drifts and saccadic eye movements. J Opt Soc Am 46:987-993. CrossRef Medline

Dash S, Dicke PW, Thier P (2013) A vermal Purkinje cell simple spike population response encodes the changes in eye movement kinematics due to smooth pursuit adaptation. Front Syst Neurosci 7:3. CrossRef Medline

Goffart L, Chen LL, Sparks DL (2004) Deficits in saccades and fixation during muscimol inactivation of the caudal fastigial nucleus in the rhesus monkey. J Neurophysiol 92:3351-3367. CrossRef Medline

Golla H, Tziridis K, Haarmeier T, Catz N, Barash S, Thier P (2008) Reduced saccadic resilience and impaired saccadic adaptation due to cerebellar disease. Eur J Neurosci 27:132-144. CrossRef Medline

Guerrasio L, Quinet J, Büttner U, Goffart L (2010) Fastigial oculomotor region and the control of foveation during fixation. J Neurophysiol 103: 1988-2001. CrossRef Medline

Guthrie BL, Porter JD, Sparks DL (1983) Corollary discharge provides accurate eye position information to the oculomotor system. Science 221: 1193-1195. CrossRef Medline

Hafed ZM (2011) Mechanisms for generating and compensating for the smallest possible saccades. Eur J Neurosci 33:2101-2113. CrossRef Medline

Hafed ZM, Krauzlis RJ (2012) Similarity of superior colliculus involvement in microsaccade and saccade generation. J Neurophysiol 107:1904-1916. CrossRef Medline

Hafed ZM, Goffart L, Krauzlis RJ (2009) A neural mechanism for microsaccade generation in the primate superior colliculus. Science 323:940-943. CrossRef Medline

Heiney SA, Kim J, Augustine GJ, Medina JF (2014) Precise control of movement kinematics by pptogenetic inhibition of Purkinje cell activity. J Neurosci 34:2321-2330. CrossRef Medline

Ignashchenkova A, Dash S, Dicke PW, Haarmeier T, Glickstein M, Thier P (2009) Normal spatial attention but impaired saccades and visual motion perception after lesions of the monkey cerebellum. J Neurophysiol 102:3156-3168. CrossRef Medline

Judge SJ, Richmond BJ, Chu FC (1980) Implantation of magnetic search coils for measurement of eye position: an improved method. Vision Res 20:535-538. CrossRef Medline

Keller EL, Robinson DA (1971) Absence of a stretch reflex in extraocular muscles of the monkey. J Neurophysiol 34:908-919. Medline

Ko HK, Poletti M, Rucci M (2010) Microsaccades precisely relocate gaze in a high visual acuity task. Nat Neurosci 13:1549-1553. CrossRef Medline

Kowler E, Steinman RM (1980) Small saccades serve no useful purpose: reply to a letter by R. W. Ditchburn. Vision Res 20:273-276. CrossRef Medline

Latour PL (1962) Visual threshold during eye movements. Vision Res 2:261-262. CrossRef

Martinez-Conde S, Otero-Millan J, Macknik SL (2013) The impact of microsaccades on vision: towards a unified theory of saccadic function. Nat Rev Neurosci 14:83-96. Medline

May PJ, Hartwich-Young R, Nelson J, Sparks DL, Porter JD (1990) Cerebellotectal pathways in the macaque: implications for collicular generation of saccades. Neuroscience 36:305-324. CrossRef Medline

Noda H, Sugita S, Ikeda Y (1990) Afferent and efferent connections of the oculomotor region of the fastigial nucleus in the macaque monkey. J Comp Neurol 302:330-348. CrossRef Medline

Ohtsuka K, Noda H (1995) Discharge properties of Purkinje cells in the oculomotor vermis during visually guided saccades in the macaque monkey. J Neurophysiol 74:1828-1840. Medline

Optican LM, Robinson DA (1980) Cerebellar-dependent adaptive control of primate saccadic system. J Neurophysiol 44:1058-1076. Medline

Otero-Millan J, Serra A, Leigh RJ, Troncoso XG, Macknik SL, MartinezConde S (2011) Distinctive features of saccadic intrusions and microsaccades in progressive supranuclear palsy. J Neurosci 31:4379-4387. CrossRef Medline

Poletti M, Rucci M (2010) Eye movements under various conditions of image fading. J Vis 10:6.1-18. Medline

Poletti M, Listorti C, Rucci M (2013) Microscopic eye movements compen- 
sate for nonhomogeneous vision within the fovea. Curr Biol 23:16911695. CrossRef Medline

Putnam NM, Hofer HJ, Doble N, Chen L, Carroll J, Williams DR (2005) The locus of fixation and the foveal cone mosaic. J Vis 5:632-639. Medline

Quinet J, Goffart L (2005) Saccade dysmetria in head-unrestrained gaze shifts after muscimol inactivation of the caudal fastigial nucleus in the monkey. J Neurophysiol 93:2343-2349. Medline

Ritchie L (1976) Effects of cerebellar lesions on saccadic eye movements. J Neurophysiol 39:1246-1256. Medline

Robinson FR, Straube A, Fuchs AF (1993) Role of the caudal fastigial nucleus in saccade generation. II. Effects of muscimol inactivation. J Neurophysiol 70:1741-1758. Medline

Sato H, Noda H (1992) Posterior vermal Purkinje cells in macaques responding during saccades, smooth pursuit, chair rotation and/or optokinetic stimulation. Neurosci Res 12:583-595. CrossRef Medline

Snodderly DM, Kagan I, Gur M (2001) Selective activation of visual cortex neurons by fixational eye movements: implications for neural coding. Vis Neurosci 18:259-277. CrossRef Medline

Steinman RM, Haddad GM, Skavenski AA, Wyman D (1973) Miniature eye movement. Science 181:810-819. CrossRef Medline
Takagi M, Zee DS, Tamargo RJ (1998) Effects of lesions of the oculomotor vermis on eye movements in primate: saccades. J Neurophysiol 80:19111931. Medline

Thier P, Erickson RG (1992) Responses of visual-tracking neurons from cortical area MST-I to visual, eye and head motion. Eur J Neurosci 4:539 553. CrossRef Medline

Thier P, Dicke PW, Haas R, Barash S (2000) Encoding of movement time by populations of cerebellar Purkinje cells. Nature 405:72-76. CrossRef Medline

Van Gisbergen JA, Robinson DA, Gielen S (1981) A quantitative analysis of generation of saccadic eye movements by burst neurons. J Neurophysiol 45:417-442. Medline

Van Horn MR, Cullen KE (2012) Coding of microsaccades in threedimensional space by premotor saccadic neurons. J Neurosci 32:19741980. CrossRef Medline

Winterson BJ, Collewijn H (1976) Microsaccades during finely guided visuomotor tasks. Vision Res 16:1387-1390. CrossRef Medline

Yamada J, Noda H (1987) Afferent and efferent connections of the oculomotor cerebellar vermis in the macaque monkey. J Comp Neurol 265: 224-241. CrossRef Medline 